\title{
Análise de Zonas Térmicas em Data Center Não-CRAC
}

\author{
Ademir Camillo Junior, Charles C. Miers, Guilherme P. Koslovski, Maurício A. Pillon \\ ${ }^{1}$ Programa de Pós-Graduação em Computação Aplicada (LabP2D/PPGCA - DCC) \\ Universidade do Estado de Santa Catarina (UDESC) - Joinville/SC - Brasil \\ ademir.juniordedu.udesc.br, \\ \{mauricio.pillon, charles.miers, guilherme.koslovski\}@udesc.br
}

Resumo. A elevada concentração de equipamentos em Data Centers (DCs) é objeto de estudo para administradores, fabricantes (processadores, servidores e sistemas de refrigeração), entre outros. Dentre os desafios da área, destaca-se o melhoramento da eficiência energética destes ambientes. No contexto de DC, a Power Usage Effectiveness (PUE) é uma referência na mensuração da eficiência energética. Este trabalho apresenta a arquitetura MonTerDC, um sistema de monitoração de temperatura de DC baseado em sistemas de refrigeração não-CRAC. Como resultados, o trabalha apresenta o uso do MonTerDC na identificação de zonas térmicas indesejáveis (fora da norma). Com este mapeamento térmico em zonas, o administrador do DC pode aplicar práticas à melhor distribuição física dos nós de computação e, consequentemente, reduzir a temperatura das zonas térmicas.

\section{Introdução}

O fornecimento crescente de serviços de Tecnologia da Informação (TI) gera a necessidade de incremento de poder computacional, atualmente, concentrados em DCs. Independente do número de equipamentos compondo um DC (pequeno, médio ou grande porte), o gerenciamento energético é um desafio recorrente. Pesquisas indicam que aproximadamente 1,3\% de toda a energia elétrica gerada atualmente é consumida por DCs [Song et al. 2015]. Esse valor é composto, principalmente por dois elementos: infraestrutura de TI, representando 52\%, e sistemas de suporte, constituindo 48\% [Ahuja et al. 2011]. Dentre os componentes dos sistemas de suporte, a climatização representa aproximadamente $39 \%$ desta fatia e afeta diretamente os custos de manutenção [Song et al. 2015].

Além de quantificar o consumo energético por categoria, determinar uma métrica que considere a relação entre os componentes de um DC, é essencial para quantificar a eficiência energética destes ambientes. O consórcio Green Grid ${ }^{1}$ estabeleceu métricas e políticas tanto para reduzir o consumo energético quanto para aumentar a eficiência energética. A métrica PUE é uma referência neste contexto e pode ser obtida pela razão entre o consumo total do DC e o consumo total dos equipamentos de TI. Quanto menor for o PUE, mais eficiente é o DC [Avelar et al. 2012]. Recentemente, DCs de grande porte divulgaram valores das suas médias trimestrais de PUEs, e.g., Google e Facebook informaram 1,10 e 1,07, respectivamente, como melhores casos [Horner and Azevedo 2016]. Por outro lado, a média mundial em 2014 foi de aproximadamente 1,7 [Sverdlik 2014].

O consumo de energia dos equipamentos de TI e sistemas de suporte podem variar, segundo as condições de temperatura do ambiente, afetando o grau de eficiência do

\footnotetext{
${ }^{1}$ https://www.thegreengrid.org/
} 
DC mensurado pelo PUE. Um dos componentes do sistema de suporte mais afetado com a variação de temperatura do ambiente é o sistema de refrigeração. No que se refere a temperatura externa, algumas organizações decidiram implantar seus DC em regiões com temperaturas médias anuais inferiores as médias globais, embora estes ambientes fiquem geograficamente distantes de seus clientes [Google 2012] [Facebook 2017].

Por outro lado, a temperatura interna de DCs sofre influência de outros fatores, tais como o projeto de sistema de refrigeração, a localização física dos equipamentos de processamento e de rede, ou ainda da carga de trabalho dos equipamentos de TI em geral. DCs de médio e grande portes são, normalmente, implantados de acordo com projetos complexos e específicos de alvenaria, elétrica e TI. Estes ambientes, em sua maioria, são climatizados por Computer Room Air Conditioner (CRAC), nos quais o ar frio é injetado por baixo, através de piso elevado perfurado, conduzido por dentro de racks de servidores, principal fonte geradora de calor em um DC, e expelido pela parte superior. $\mathrm{O}$ controle do fluxo de ar frio direcionado a fontes de geração de calor torna o modelo CRAC eficiente [Arghode and Joshi 2013], entretanto, exigem um elevado custo de implantação, se comparados com sistemas simples. Em resumo, o custo de implementação é um dos principais fatores para baixa utilização em DCs de pequeno e médio porte. No Brasil, apenas 8\% dos DCs utilizam CRAC [Schneider 2014].

Neste trabalho, DCs constituídos de sistemas de refrigeração formados por condicionadores de ar e poucos pontos de injeção de ar, se comparado com CRAC que possui algumas dezenas de entradas/saídas direcionadas e projeto de condução pressurizada das correntes de ar frio e quente, são nomeados de não-CRAC. Sistemas de refrigeração não-CRAC, maioria dos DCs brasileiros, são mais suscetíveis a má distribuição das correntes de ar e a formação de zonas térmicas indesejáveis, não respeitando as normas de padronização [ASHRAE 2016] [Fulpagare et al. 2016].

Alguns fatores que podem influenciar na formação de zonas térmicas quentes que excedam as especificadas nas normas, são: a má distribuição físicas dos equipamentos de TI, a ausência de condução das correntes de ar frio / quente ou a variação da carga de processamento dos equipamentos. Os dois primeiros fatores referem-se ao projeto físico de concepção do DC, são fatores estáticos, pois o administrador não costuma deslocar seus servidores após a instalação física e tampouco direcionar as aletas do condicionador de ar de acordo com a carga do DC. O último fator, variação da carga de processamento, pode ser considerado pelo administrador no momento de provisionamento de recursos ou balanceamento de carga. Neste caso, o administrador define o servidor hospedeiro de acordo com a necessidade de processamento solicitada e a distribuição térmica atual entre as zonas. Portanto, ao provisionar um novo recurso, ele pode escolher um servidor $X$, localizado em uma zona $Y$, em detrimento a um servidor $W$, de igual poder computacional, localizado em uma zona $Z$, pelo fato da zona $Z$ ter temperatura mais elevada do que a zona $Y$. Desta forma o equilíbrio térmico do DC é mantido.

O monitoramento térmico em tempo real é uma importante ferramenta para auxiliar a tomada de decisão do administrador do DC. A arquitetura Monitoramento Término de Data Centers (MonTerDC), proposta deste trabalho, é constituída de três módulos independentes e pode ser integrada a diversos frameworks gerenciadores. A MonTerDC foca em ambientes de DC de pequeno e médio porte, sem sistemas de refrigeração complexos que seguem o modelo CRAC. Os resultados da monitoração das zonas térmicas 
no DC do LabP2D, um DC de pequeno porte e uma única fonte de ar frio, permitem quantificar o impacto do provisionamento de recursos na formação de zonas indesejáveis. Com o equilíbrio térmico no ambiente, evitando zonas térmicas indesejáveis, o sistema de refrigeração é menos exigido e, consequentemente, o consumo energético total do DC é reduzido.

Este artigo está organizado em quatro seções. Trabalhos correlatos são discutidos na Seção 2. A arquitetura MonTerDC é apresentada na Seção 3, com a descrição dos componentes e da ferramenta para monitoração de zonas térmicas. A Seção 4 apresenta os experimentos, descrevendo o cenário, o método de testes e a análise dos resultados coletados. A Seção 5 finaliza o trabalho e apresenta perspectivas para continuação.

\section{Trabalhos correlatos}

O monitoramento térmico é uma prática reconhecida em DCs de grande porte, cujo sistema de refrigeração segue o modelo CRAC. A Tabela 1 apresenta a comparação entre os trabalhos relacionados. Dentre eles, as soluções diferem quanto ao objetivo, a aplicação e ao componente observado. Alguns trabalhos observam o comportamento do processador, do servidor, do rack ou da totalidade do DC.

\begin{tabular}{c|c|c|c|c}
\hline \hline Autor & Nível & Coleta de dados & Aplicação & Objetivo \\
\hline \hline [Tang et al. 2008] & data center & modelo / CFD & simulação & circulação de ar \\
\hline [Pakbaznia et al. 2010] & data center & - & real & balanceamento de carga \\
\hline [Lei et al. 2011] & rack & sensoriamento & real & predição \\
\hline [Ahuja et al. 2011] & rack & CFD & simulação & contenção no rack \\
\hline [Alkharabsheh et al. 2014] & data center & modelo / CFD & simulação & saída de ar quente \\
\hline [Bottari 2014] & data center & sensoriamento & real & predição / prevenção \\
\hline [Wibron 2015] & data center & CFD & simulação & localização física \\
\hline [Zhang et al. 2015] & data center & sensoriamento & real & pressão do ar \\
\hline [Gao et al. 2016] & rack & - & real & fluxo de ar \\
\hline \hline
\end{tabular}

Tabela 1. Trabalhos relacionados cronologicamente ordenados.

Com relação ao nível de atuação do monitoramento e refrigeração pode-se identificar soluções a nível de rack ou para o DC como um todo. Gao et al. 2016 abordam o comportamento do fluxo de ar internamente ao rack através de um experimento real, entretanto, não se preocupam com o posicionamento dos equipamentos e como a localização interna afeta o aquecimento. Lei et al. 2011 fazem uma análise semelhante, utilizando sensores para realizar uma predição de cenários em tempo real. Por outro lado, através de simulação, Ahuja et al. 2011 analisam o fluxo do ar internamente ao rack utilizando Computational Fluid Dynamics (CFD), porém não aborda as diferentes cargas de trabalho em diversos cenários.

Outro conjunto de trabalhos busca fornecer informações, através de simulações, para ambientes de DCs controlados. Neste caso, o DC não necessariamente está operacional, portanto, pode ser interessante para a concepção de projetos de DC. A simulação através da Dinâmica dos Fluidos Computacionais, CFD é utilizada para prever as velocidades dos fluídos e suas respectivas temperaturas ou para modelar as correntes de ar 
frio/quente em ambientes de DC [Marshall and Bemis 2011, Wibron 2015]. Através de simulações, Ahuja et al. 2011 indicou como utilizar o CFD para analisar um ambiente de DC de alta disponibilidade para identificar a diferença de temperatura quando é utilizado contenção nos racks, evitando que o ar quente re-circule no DC. Por sua vez, Tang et al. 2008 apresentou um modelo de circulação de ar para DC visando diminuir o custo com climatização, diminuindo entre 2 e 5 graus a temperatura média, com economia de $20 \%$ a $30 \%$ no consumo de energia. Entretanto, ambos não relacionaram as cargas de trabalho dinâmicas que ocorrem nestes ambientes de alto desempenho. De forma complementar, [Alkharabsheh et al. 2014] utilizou CFD em um DC para prever a temperatura de saída do ar quente para ajustar o sistema de refrigeração.

Analisando o método de coleta dos dados, quando observada a utilização de sensores, [Lei et al. 2011], propuseram o ThermoCast, uma solução de monitoramento com sensores que realiza o mapeamento de zonas térmicas em um rack, para identificar as zonas de calor e predizer o superaquecimento individualizado em cada estrutura. Entretanto, nesta solução, uma visão macro do ambiente não é possível, atendo-se apenas ao nível de rack. Também utilizando sensores, Bottari 2014 apresenta uma arquitetura para monitoração de DCs através de Internet of Things (IoT), com objetivo de identificar e prever o superaquecimento, porém, não se preocupando com as zonas de calor ou a influência entre elas.

Por outro lado, para gerenciar a temperatura do ar frio injetado, Zhang et al. 2015 apresentam um sistema para utilização em CRAC, que através da coleta de informações de temperatura em tempo real e mapeamento de zonas, permite regular a quantidade de ar frio injetado no sistema em cada área gerenciada. Ainda, Gao et al. 2016 propõem uma unidade de refrigeração e circulação de ar individualizada para cada rack que permite a coleta do ar frio do sistema de ventilação CRAC e envio direto ao rack, gerando um fluxo de ar frio específico para cada área.

Como alternativa para a redução do consumo de energia, pode-se realizar a migração de máquinas virtuais para outros servidores ou locais físicos. Em [Pakbaznia et al. 2010] o objetivo é a redução de energia através do balanceamento de carga, distribuindo processos entre vários servidores e mantendo suas cargas equivalentes. A técnica utilizada por [Alkharabsheh et al. 2014] segue a lógica oposta, utiliza-se da consolidação de processos no menor número de servidores possível. Ambos convergem no ambiente de aplicação, DCs de produção e CRAC, entretanto, não se preocupam com as novas zonas térmicas originadas pelo balanceamento das cargas e variação da temperatura.

Por fim, é importante ressaltar que os trabalhos relacionados focam em ambientes CRAC, sobretudo na pressão do ar injetado no sistema com objetivo de otimização do resfriamento e menor consumo de energia, mas não discutem os locais que precisam um maior fluxo de ar frio devido a uma temperatura maior e variável. Outro aspecto relevante é o foco no consumo de energia dos equipamentos de TI, sem preocupação dedicada ao consumo de energia com climatização, diretamente influenciado pelas zonas de calor geradas pelo aquecimento dos equipamentos de TI que estão sendo gerenciados.

\section{Monitoramento Térmico de data center não-CRAC (MonTerDC)}

A concentração de potência de cálculo em DCs é uma realidade. A monitoração destes ambientes é essencial tanto para administradores quanto para usuários que buscam 
eficiência no uso dos recursos em prol de suas aplicações ou, simplesmente, otimização de custos. Neste contexto, este trabalho descreve uma proposta de arquitetura para um ambiente de monitoração de DCs de pequeno e médio porte. A proposta de arquitetura do MonTerDC, ambiente de Monitoramento Térmico de data centers, tem por objetivo fornecer um mapa térmico dinâmico em tempo real de DCs não-CRAC. Este ambiente consiste da definição de módulos de hardware e de software que, integrados, permitem a identificação e mapeamento de zonas térmicas.

As zonas térmicas podem ser formadas por uma ou mais fontes geradoras de ar frio ou quentes e não possuem dimensões máximas ou mínimas. Em ambientes de DCs com sistemas de refrigeração não-CRAC, as correntes de ar frio e quente não são guiadas por projetos de canaletas, tubos, pisos elevados ou injeção de ar. A formação de zonas indesejáveis, que excedam as temperaturas máximas especificadas nas normas, são afetadas pela intensidade do gerador de calor ou pelo número de geradores concentrados, pela pressão da injeção de ar e pela umidade. Nestes ambientes, onde o controle dos fatores que influenciam na formação de zonas indesejáveis não são controlados, a identificação de zonas térmicas indesejáveis torna-se mais complexa. A característica dinâmica dos fatores, o deslocamento das zonas e a interferência entre elas dificultam ainda mais a identificação. Esta realidade é específica de DCs com sistemas de regrigeração não-CRAC, pois as zonas térmicas em DCs CRAC são bem definidas e controladas, com temperaturas baixas na parte inferior e quentes superiores, variando apenas os valores de acordo com a carga de trabalho dos servidores ativos [Athavale et al. 2016].

A arquitetura do MonTerDC é constituída de três módulos principais: Central de Coleta (C), Central de Processamento (CP) e o 3D Viewer. A relação entre estes módulos está ilustrada na Figura 1. O primeiro módulo, a Central de Coleta é composta de sensores especializados na captura dos fatores externos de formação das zonas térmicas, tais como temperatura, umidade e pressão atmosférica. Com capacidades de armazenamento e processamento limitadas, a Central de Coleta limita-se a aquisição das informações do ambiente de acordo com as especificações parametrizadas pelo administrador. O administrador define o intervalo de captura, tipo da informação, a faixa de erro tolerada nas capturas e a necessidade ou não de pré-processamento das informações. Caso o préprocessamento seja ativado, o administrador deve informar o método estatístico de sua preferência (média, mediana, etc) que deve ser aplicado a estes dados.

A Central de Processamento cabe a manipulação dos dados coletados em tempo real, associando-os a infraestrutura física do DC. Os dados individualizados por sensor são etiquetados ( $t a g$ ) e armazenados em um banco de dados. Este módulo dispõe da estrutura física do DC, localização de servidores, das saídas do sistema de refrigeração e dos sensores. Neste trabalho, uma zona térmica não tem uma dimensão física mínima ou máxima e deve ser formada por pelo menos um sensor. $\mathrm{O}$ número de sensores por zona térmica varia em função do número de fontes geradoras de calor ou frio e da precisão dos sensores. A dimensão de uma zona térmica é determinada pelo espaço físico com temperatura homogênea considerando o erro de precisão dos sensores. De posse destas informações, o submódulo Simulador CFX gera o cenário atual do ambiente de DC determinando as correntes de ar frio e quente, a intensidade destas correntes no ambiente e as zonas térmicas com suas respectivas temperaturas. 


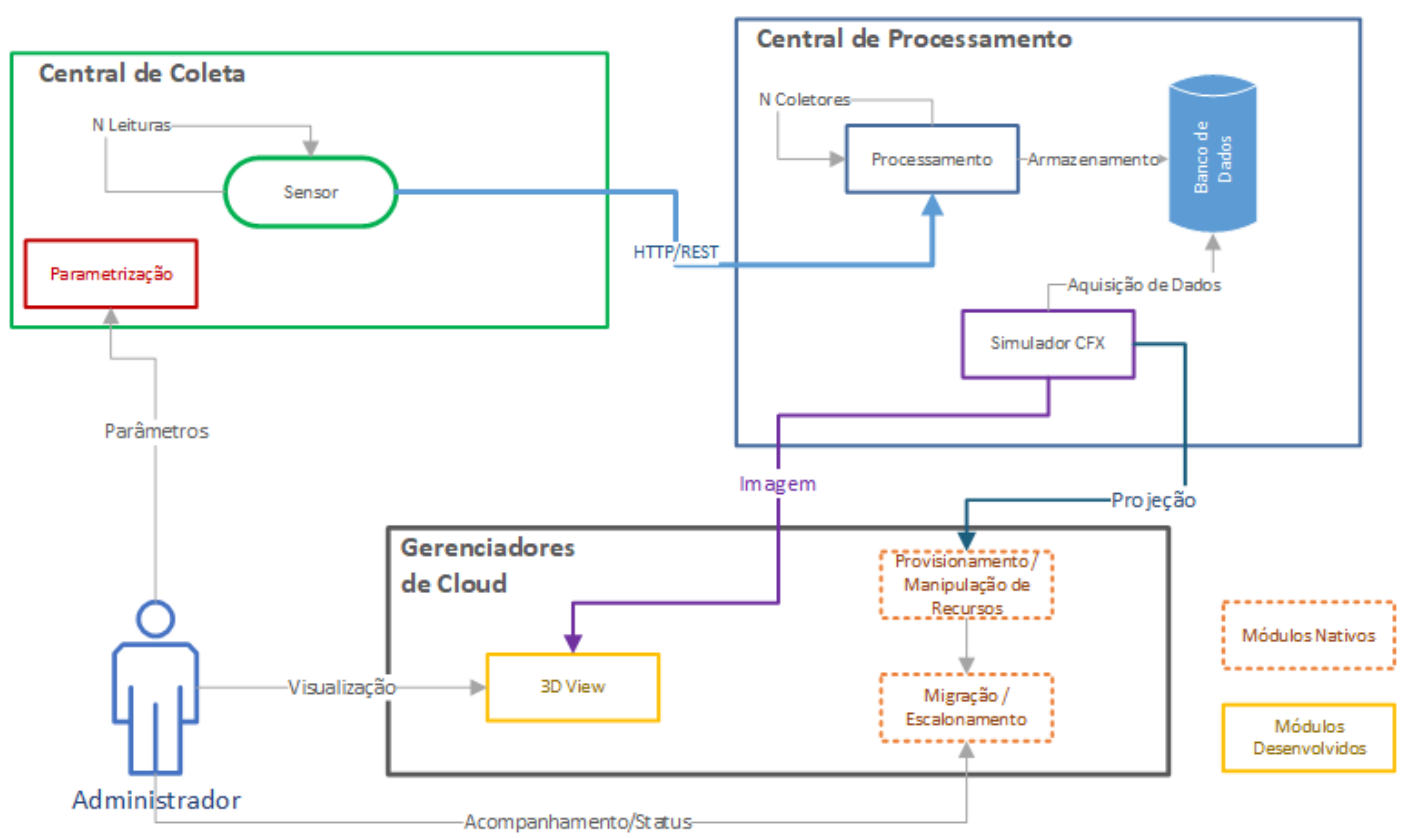

Figura 1. Arquitetura MonTerDC.

O último módulo, 3D View, é o responsável pela visualização do mapa térmico do ambiente. Ele está integrado a um gerente de recursos, de nuvem como ilustrado na Figura 1, ou de data centers. Com a visualização em tempo real no 3D View das zonas térmicas em ambiente de data center não-CRAC, a arquitetura MonTerDC auxilia o administrador de data center de pequeno e médio porte.

\section{Estudo de Caso: LabP2D}

O Laboratório de Processamento Paralelo e Distribuído (LabP2D) está vinculado a Universidade do Estado de Santa Catarina (UDESC), campus Joinville. O LabP2D possui um espaço físico no andar térreo de um prédio de três andares, originalmente projetado para acolher uma sala de aula. O ambiente possui sistema de climatização tradicional do tipo Split (Electrolux - 30.000 btus), fonte geradora fria, com uma única saída. Ele encontrase localizado na parte superior da sala e o sistema de direcionamento do fluxo de ar é feito através de aletas. Neste ambiente, o LabP2D abriga o seu DC de pequeno porte que conta atualmente com duas fileiras de máquinas servidoras do tipo torre e um rack de chão com três switches e três servidores de produção do tipo rack. Cada fila de servidores do tipo torre contam com 10 máquinas HP Proliant (processador AMD Phenom II X4 B93 2,8GHz, 4 núcleos, 8GB RAM, 500GB HD, Fonte 150W). Os servidores de produção instalados no rack possuem a seguinte configuração: 2 processadores Intel Xeon E5-2600, 24 núcleos, 292GB RAM, 2TB HD, fonte $740 \mathrm{~W}$ redundante. Atualmente, este DC hospeda a Nuvem Tche cujo o gerenciador de recursos é o OpenStack (Ocata Release). Na Figura 2, pode-se observar a modelagem do ambiente físico em 2D, representando as duas fileiras de servidores torre HP, na horizontal, e o rack com os servidores de produção e switches no meio a esquerda.

A implantação do sistema de Monitoramento Térmico de DC (MonTerDC) no LabP2D respeitou as restrições impostas pelo ambiente. Na Figura 2(a), ilustra-se o posicionamento físico dos sensores numerados de 1 a 14. Na Figura 2(b) é possível identificar 


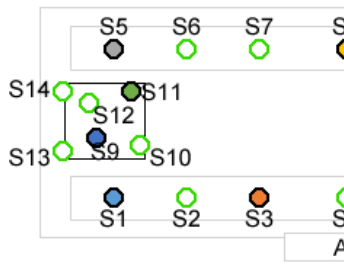

a) Localização dos Sensores

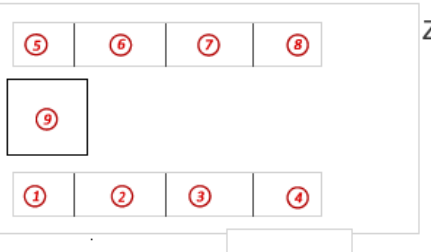

b) Blocos de Processamento

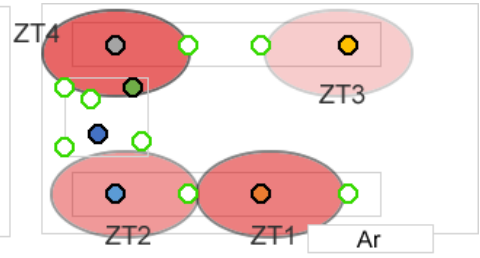

c) Área das Zonas de Testes

Figura 2. Estudo de caso LabP2D

os blocos de processamento. No caso das fileiras superior e inferior, cada bloco (de 1 à 4 e de 5 à 8) é constituído de 4 servidores HP. O bloco 9 , localizado no meio a esquerda, é constituído dos 3 servidores de produção e 3 switches. Finalmente, na Figura 2(c) estão representadas as zonas térmicas identificadas pelo MonTerDC. Os sensores de temperatura são do tipo LM35DZ, possuem precisão de $1^{\circ}$ Celsius e estão posicionados a uma distância máxima entre eles de 1 metro. Desta forma, foi possível identificar as mudanças de temperatura entre duas ou mais zonas de calor simultaneamente.

Os nós de processamento foram agrupados em blocos de processamento, com o objetivo de alternar as fontes geradoras de calor variando a carga de trabalho destas máquinas entre $0 \%$ e $100 \%$. Como o objetivo do teste é observar o impacto das fontes de calor nas zonas, definiu-se que o bloco de processamento central, número 9 formado pelo rack, permanecesse com $100 \%$ de carga de trabalho. Os quatro cenários de testes realizados seguem a descrição da Tabela 2 que varia a carga de processamento por bloco.

Tabela 2. Configuração da carga de processamento no cenário de teste.

\begin{tabular}{|l|r|r|r|r|r|}
\hline \multirow{2}{*}{ Cenário } & \multicolumn{5}{|c|}{ Carga de Processamento } \\
\cline { 2 - 6 } & Bloco 1 & Bloco 3 & Bloco 5 & Bloco 8 & Bloco 9 \\
\hline Cenário 1 & $0 \%$ & $100 \%$ & $0 \%$ & $0 \%$ & $100 \%$ \\
\hline Cenário 2 & $100 \%$ & $0 \%$ & $0 \%$ & $0 \%$ & $100 \%$ \\
\hline Cenário 3 & $0 \%$ & $0 \%$ & $0 \%$ & $100 \%$ & $100 \%$ \\
\hline Cenário 4 & $0 \%$ & $0 \%$ & $100 \%$ & $0 \%$ & $100 \%$ \\
\hline
\end{tabular}

O objetivo da variação de cenários (Tabela 2) é observar o comportamento das zonas térmicas. A geração de calor é intensa e constante no bloco 9 para todos os cenários. A escolha de intensificação e suavização alternada dos blocos 1, 3, 5 e 8 buscam identificar o comportamento das zonas nas fileiras de servidores HP. Observa-se que a posição do sistema de refrigeração favorece a circulação de ar frio em alguns blocos, por exemplo bloco 8 , não atuando na mesma forma no bloco 1 .

A bateria de testes com duração de 15 minutos cada foi aplicada a todos os cenários. Ela compreende de uma fase de aquecimento, próximo a 3 minutos e de estabilização/observação da temperatura do bloco (12 minutos). A cada nova bateria, aplica-se uma fase de resfriamento do ambiente, onde todos os blocos tem carga de $0 \%$ por 15 minutos. Finalmente, como saída do sistema (Figura 3), os dados coletados pelo ambiente MonTerDC geram figuras do ambiente de DC em 3D com o auxílio do software ANSYS CFX 17.2. 


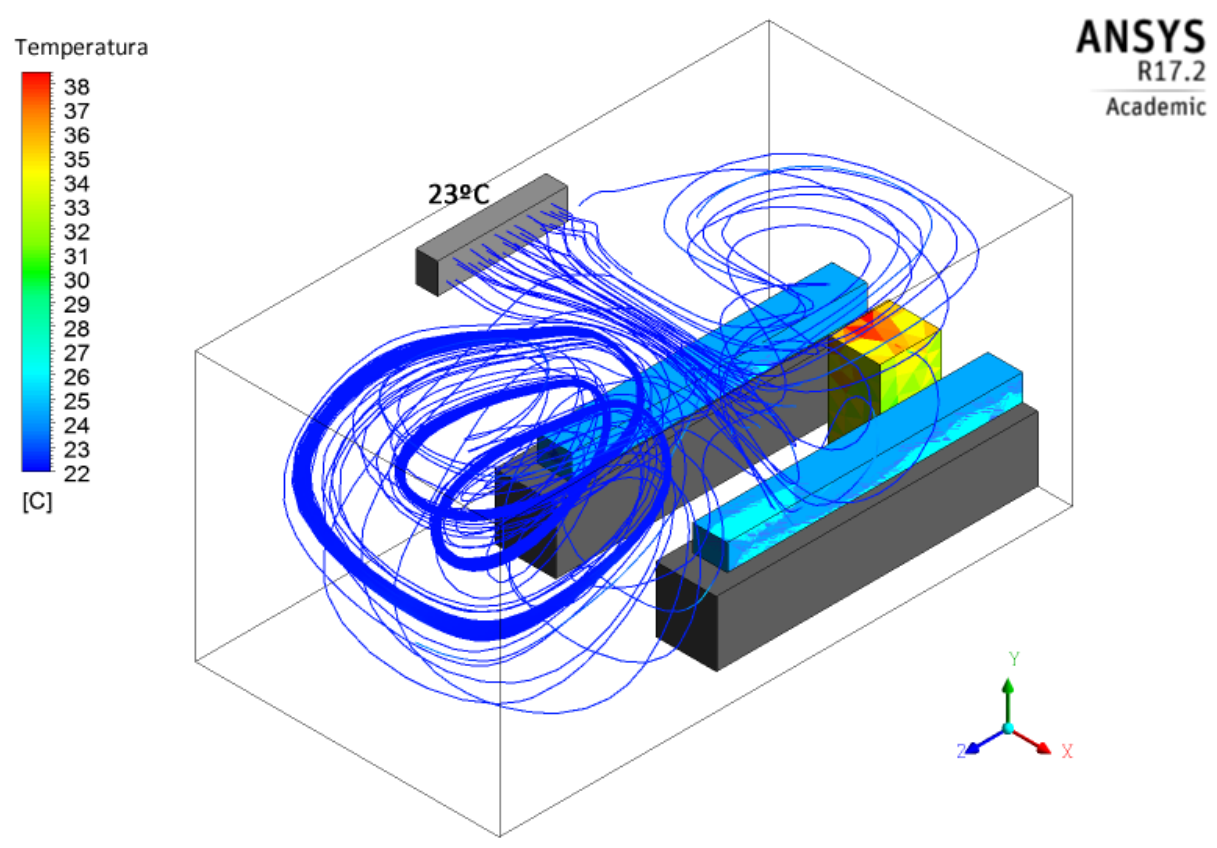

Figura 3. Resultado de saída da simulação do ambiente de testes.

Na Figura 3, tem-se um exemplo da modelagem do fluxo de corrente de ar frio do ambiente do LabP2D modelado com o software Ansys Academic 17.2. A integração do MonTerDC com o $A N S Y S$ que permite a visualização das zonas sobrepostas ainda está em fase de testes.

\section{Resultados}

Nesta seção são descritos os resultados da observação do mapeamento térmico do LabP2D, através do sistema MonTerDC, nos quatro cenários propostos. Os aspectos analisados foram: formação de zonas térmicas indesejáves, o impacto de um bloco de processamento a seus vizinhos e a diferença de comportamento das zonas térmicas entre blocos com a mesma carga, porém em localizações físicas diferentes.

A execução da carga de processamento de acordo com a definição de blocos e cenários, atuou no ambiente do LabP2D com a formação de quatro zonas de observação, uma para cada bloco de processamento envolvido. As zonas foram nomeadas de Zonas Térmicas (ZT) de ZT1 à ZT4 de acordo com os blocos, respectivamente, identificados com 3, 1, 8 e 5 (Figura 2(c)). Os resultados analisam o comportamento nas zonas formadas em função do processamento dos blocos envolvidos nos cenários e o impacto do processamento destes blocos na temperatura do bloco 9, bloco central de rack com carga constante.

A Figura 4, identifica as temperaturas obtidas nos sensores $S 1, S 3, S 5, S 8, S 9$ e $S 11$, eixo $y$, e o horário de início e térmico de cada bateria de testes, eixo $x$. A escolha dos sensores se deu em função dos objetivos da análise destes testes. Os primeiros quatro correspondem aos sensores centrais dos blocos de processamentos envolvidos e, os últimos dois, localizados no bloco 9, são os afetados pela zona de términa de seus vizinhos. A mensuração dos testes seguiu um período contínuo de observação, iniciando-se as 9:42 
e terminando as 11:38. Presume-se que as condições de temperatura, umidade e pressão externas, que podem influenciar nos resultados, são constantes para todos os cenários. Finalmente, os retângulos tracejados observados ao longo do eixo $x$ demarcam o período de aplicação dos cenários.

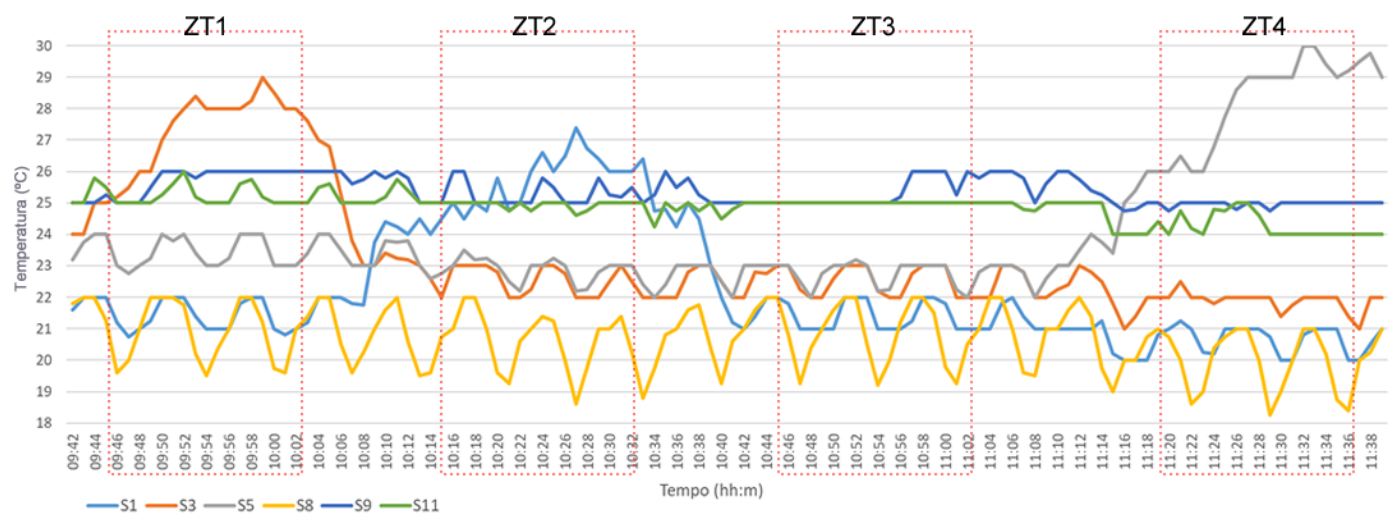

Figura 4. Mapeamento das Zonas Térmicas (ZT) no ambiente de testes.

Observando o comportamento das temperaturas (Figura 4), pode-se constatar que o impacto da carga de processamento não afeta da mesma forma todos os blocos. $\mathrm{O}$ bloco 8, medido pelo sensor $S 8$, é o menos afetado pela sua própria carga de processamento ou pela carga de seus vizinhos. A sua temperatura varia entre pouco mais de $18^{\circ} \mathrm{e}$ $22^{\circ}$, com ondulações regulares, que correspondem as oscilações do fluxo de ar do sistema de refrigeração. Observando-se as correntes de ar frio, apresentadas na Figura 3, constatase que a localização do bloco 8 recebe correntes de ar frio com maior intensidade.

O bloco de processamento 5 indica um comportamento totalmente oposto, medido pelo sensor $S 5$. A faixa de temperatura deste bloco é entre $22^{\circ}$ e $30^{\circ}$, atingindo portanto temperaturas superiores as especificadas nas duas normas abordadas. No período de repouso, com carga de processamento $0 \%$, este bloco atinge sua temperatura mínima de $22^{\circ}$ nas fases de resfriamento e nos cenários em que o fluxo de refrigeração não é afetado (ZT2 e ZT3). Porém, a ativação do bloco de processamento 3, incrementou a temperatura do bloco $5 \mathrm{em} 1^{\circ}$. Embora o bloco 3 não seja seu vizinho, a sua ativação alterou o fluxo de corrente de ar frio central que refrigera o bloco 5 . Com esta nova fonte geradora de calor, o bloco 5 já prejudicado, se comparado com os demais, foi ainda mais afetado. Finalmente, com a ativação da sua carga de processamento, o bloco 5 atingiu as temperaturas mais altas do teste, chegando a $30^{\circ}$, e formando uma zona indesejável.

Com temperaturas mais amenas, se comparadas as do bloco 5 , o comportamento dos blocos 1 (sensor $S 1$ ) e 3 (sensor $S 3$ ) são semelhantes ao do bloco 5 . As temperaturas do bloco 1 variam entre $21^{\circ}$ e pouco mais de $27^{\circ}$ e as do bloco 3 entre $21^{\circ}$ e $29^{\circ}$, ambos formando zonas indesejáveis quando a carga de processamento é aplicada a seu respectivos blocos. No entanto, dadas as suas localizações físicas e a distribuição das correntes de ar frio, eles não são afetados por nenhuma outra fonte geradora de calor, mas afetam outras. A única diferença observada entre os blocos 1 e 3 é que o bloco 1 afeta a zona térmica de seu vizinho direto (bloco 9). Já o bloco 3 alterou o fluxo da corrente de ar influenciando a temperatura do bloco 5 . 
Os últimos dois sensores, $S 9$ e $S 11$, monitoraram o bloco 9 cujo a carga de processamento não variou. As temperaturas neste bloco tiveram pouca variação, ficando entre $24^{\circ}$ e $26^{\circ}$. No entanto, suas maiores temperaturas, mesmo dentro da norma, ocorreram quando o bloco 1, vizinho direto, foi ativado ou quando o bloco 8, distante físicamente, ao intensificar sua geração de calor, afetou a corrente de ar frio que chegava ao bloco 9 .

A aplicação do ambiente MonTerDC ao estudo de caso do LabP2D, um DC de pequeno porte não-CRAC, revelou a importância da proposta deste trabalho na identificação da formação dinâmica das zonas térmicas. A formação de zonas térmicas indesejáveis, ou mesmo de zonas térmicas dentro da norma mas com temperaturas altas, tem influência direta no consumo de energia e na durabilidade dos equipamentos de TI e sistemas de suporte. A análise dos resultados, através do MonTerDC, permitiram identificar as zonas térmicas visualmente e, principalmente, os fatores de interferências entre estas zonas. Normalmente, a influência térmica entre vizinhos é considerado por administradores, porém a alteração do fluxo de refrigeração, devido a intensificação ou suavização de fontes geradoras de calor, é um fator mais complexo de identificar sem o auxílio de ferramentas como o MonTerDC.

\section{Considerações \& Trabalhos futuros}

A concentração de equipamentos de TI em DCs facilita a gerência destes recursos, contando com equipes especializadas e infraestrutura dedicada. Porém, o comportamento dos equipamentos de TI varia conforme a carga de processamento e é influenciado por fatores, tais como: temperatura, pressão e umidade. A monitoração destes fatores em tempo real, por si só, já é um desafio, mas compreender a relação entre eles não é trivial. Para o administrador, entender esta relação é essencial, pois a simples decisão de alocar um bloco de processamento em uma zona $A$ pode afetar diretamente a temperatura da zona vizinha $B$ ou a corrente de ar que refrigera a zona $C$. Enfim, estas relações são dinâmicas, não necessariamente evidentes e impactam no consumo de energia total do DC e a vida útil dos equipamentos.

Este trabalho apresentou a arquitetura do MonTerDC, um sistema de Monitoramento Térmico para data centers de pequeno e médio porte não-CRAC. Embora, DCs com sistemas de refrigeração não-CRAC sejam a realidade de $\sim 92 \%$ dos DCs implantados no Brasil [Schneider 2014], poucos trabalhos científicos tratam do problema. O MonTerDC apoia-se em uma malha de sensores de temperatura, vinculando a estrutura física estática, localização dos equipamentos e a natureza dinâmica do fluxo de refrigeração formado por geradores de correntes de ar frio e quente. Com estas informações, o MonTerDC modela as zonas térmicas, identificando zonas indesejáveis (com temperaturas superiores as especificadas nas normas) e, através de um simulador de CFD, gera uma imagem 3D com as zonas térmicas em tempo real.

Os resultados reforçam a importância de ambientes de monitoração térmica neste contexto. Comprovou-se a influência entre vizinhos, por exemplo, a variação da carga de processamento do bloco 1 afetou a temperatura do bloco 9, mas também identificou-se o impacto no fluxo de ar frio gerado pela variação da carga do bloco 8 ou do bloco 3 . A alteração do fluxo de ar frio afetou a temperatura tanto do bloco 9 quanto do bloco 5 . Embora os blocos de processamento das fileiras serem formados pelo mesmo número de máquinas, no caso 4 servidores de torre $\mathrm{HP}$, e terem a mesma carga de processamento, 
ora $0 \%$ ora $100 \%$, as zonas térmicas formadas em seus entornos possuem diferenças de até $7^{\circ} C$. Observou-se claramente a existência de blocos suscetíveis às variações de cargas de outros blocos, tais como blocos $1,3,5$ e 9 , e outros, no caso dos testes, somente o bloco 8, totalmente indiferente a atuação dos demais. O protótipo da arquitetura MonTerDC está operacional e mostrou a sua importância na identificação de zonas térmicas no contexto a que se propõe. No âmbito do protótipo, os principais pontos de melhora focam na integração do MonTerDC ao gerente de recursos em nuvem OpenStack e na automatização do módulo de simulação CFX.

\section{Agradecimentos}

Os autores agradecem o apoio do Laboratório de Processamento Paralelo e Distribuído (LabP2D) no Centro de Ciências Tecnológicas (CCT) da Universidade do Estado de Santa Catarina (UDESC) e a Fundação de Amparo à Pesquisa e Inovação do Estado de Santa Catarina (FAPESC).

\section{Referências}

Ahuja, N., Rego, C., Ahuja, S., Warner, M., and Docca, A. (2011). Data center efficiency with higher ambient temperatures and optimized cooling control. In 2011 27th Annual IEEE Semiconductor Thermal Measurement and Management Symposium, pages 105109.

Alkharabsheh, S., Sammakia, B., Shrivastava, S., and Schmidt, R. (2014). Implementing rack thermal capacity in a room level CFD model of a data center. In 2014 Semiconductor Thermal Measurement and Management Symposium (SEMI-THERM), pages 188-192.

Arghode, V. K. and Joshi, Y. (2013). Modeling Strategies for Air Flow Through Perforated Tiles in a Data Center. IEEE Transactions on Components, Packaging and Manufacturing Technology, 3(5):800-810.

ASHRAE (2016). Ashrae tc9.9, data center networking equipment - issues and best practices.

Athavale, J., Joshi, Y., Yoda, M., and Phelps, W. (2016). Impact of active tiles on data center flow and temperature distribution. In 2016 15th IEEE Intersociety Conference on Thermal and Thermomechanical Phenomena in Electronic Systems (ITherm), pages 1162-1171.

Avelar, V., Azevedo, D., and French, A. (2012). PUE ${ }^{\mathrm{TM}}$ : A Comprehensive examination of the metric.

Bottari, G. D. (2014). Monitoramento Térmico Responsivo para Centros de Processamento de Dados. Master Degree, Universidade Federal Fluminense, Niteroi/RJ - Brasil.

Facebook (2017). Lulea datacenter. In https://www.facebook.com/LuleaDataCenter.

Fulpagare, Y., Shirbhate, P., and Bhargav, A. (2016). Design and testing of prototype data center. In 15th IEEE ITHERM Conference, Indian Institute of Technology Gandhinagar. 
Gao, T., Kumar, E., Sahini, M., Ingalz, C., Heydari, A., Lu, W., and Sun, X. (2016). Innovative server rack design with bottom located cooling unit. In 2016 15th IEEE Intersociety Conference on Thermal and Thermomechanical Phenomena in Electronic Systems (ITherm), pages 1172-1181.

Google (2012). Google datacenters - from paper mill to data center. In https://www.google.com/about/datacenters/inside/locations/hamina/.

Horner, N. and Azevedo, I. (2016). Power usage effectiveness in data centers: Overloaded and underachieving. In The Electricity Journal - 29, pages 61-69.

Lei, L., Liang, C., and Liu, J. (2011). Thermocast: A cyber-physical forecasting model for data centers. In 17th ACM SIGKDD Conference on Knowledge Discovery, San Diego, California, USA.

Marshall, L. and Bemis, P. (2011). Using CFD for data center design and analysis. Applied Math Modeling White Paper.

Pakbaznia, E., Ghasemazar, M., and Pedram, M. (2010). Temperature-aware dynamic resource provisioning in a power-optimized datacenter. In 2010 Design, Automation Test in Europe Conference Exhibition (DATE 2010), pages 124-129.

Schneider, E. (2014). Soluções em climatização para data center. Brasília/Brasil. XIV Encontro Nacional de Empresas Projetistas e Consultores da Abrava.

Song, Z., Zhang, X., and Eriksson, C. (2015). Data Center Energy and Cost Saving Evaluation. Energy Procedia, 75:1255-1260.

Sverdlik, Y. (2014). Survey: Industry average data center pue stays nearly flat over four years. Uptime Institute.

Tang, Q., Gupta, S. K. S., and Varsamopoulos, G. (2008). Energy-Efficient ThermalAware Task Scheduling for Homogeneous High-Performance Computing Data Centers: A Cyber-Physical Approach. IEEE Transactions on Parallel and Distributed Systems, 19(11):1458-1472.

Wibron, E. (2015). CFD Modeling of an Air-Cooled Data Center. Master Degree, CHALMERS University of Technology, Gothenburg/Sweden.

Zhang, S., Liu, X., Ahuja, N., Han, Y., Liu, L., Liu, S., and Shen, Y. (2015). On demand cooling with real time thermal information. In 2015 31st Thermal Measurement, Modeling Management Symposium (SEMI-THERM), pages 138-146. 